\title{
Implantação da farmácia viva na UNEF para o uso nos atletas de futebol juvenil do Bahia de Feira - BA
}

\author{
Wilma Brandão de Andrade ${ }^{\mathrm{a}}$, Denise da Silva Araújo a , José Luiz Carneiro da Rocha ${ }^{\mathrm{a}, \mathrm{b}^{*}}$
}

aLiga Acadêmica de Farmácia Social e Clínica (LAFSC) e Curso Farmácia da Unidade de Ensino Superior de Feira de Santana - UNEF, Feira de Santana, BA, Brasil

${ }^{\mathrm{b}}$ Liga Acadêmica de Fitoterapia, Estética e Dermatologia (LAFEDERM) e Unidade de Ensino Superior de Feira de Santana - UNEF, Feira de Santana, BA, Brasil

Histórico do Artigo Recebido em: 30/10/2019

Aceito em: $23 / 03 / 2020$

Palavras-chave: Fitoterapia; jogadores de futebol; plantas medicinais; mandala terapêutica

Keywords:

Medicinal plants; Phytotherapy; Soccer players; Mandala Therapeutic

\begin{abstract}
RESUMO
O referente trabalho trata da implantação da farmácia viva na Unidade de Ensino Superior de Feira de Santana - UNEF com a finalidade do uso de plantas medicinais com características terapêuticas relacionadas às atividades esportivas dos jogadores juvenis de futebol, tanto para fins preventivos quanto curativos. A farmácia viva permite aplicar a cultura popular e científica do uso das plantas no tratamento de pequenos agravos ou manutenção da saúde que acontecem nesse tipo de modalidade desportiva. Para a implantação, foi construída uma estrutura de madeira em forma de uma mandala com formato octogonal para o plantio das 8 espécies selecionadas: alecrim (Rosmarinus officinalis L.), arnica (Porophyllum ruderale (Jacq.) Cass.), babosa (Aloe vera (L.) Burm.f), calêndula (Calendula officinalis L.), chicória (Cichorium intybus L.), mastruz (Dysphania ambrosioides (L.) Mosyakin e Clemants), tanchagem (Plantago major L.) e terramicina (Alternanthera brasiliana (L.) Kuntze), sendo dispostas em formato triangular para cada espécime e depositadas na Horta "Green UNEF" para o cultivo com a finalidade do uso nos atletas de acordo com suas necessidades desportivas. Assim, ao longo dessa iniciativa, a implantação da farmácia viva mostrou ser um sistema com grande viabilidade para a modalidade desportiva desde o cultivo até a utilização de plantas medicinais que auxiliam na manutenção da saúde ou prevenção de pequenos agravos, como também, por ser uma alternativa de terapia medicamentosa a partir de fitoterápicos, que permite consequentemente a redução de gastos com medicamentos sintéticos.
\end{abstract}

Implementation of the living pharmacy at UNEF for use in youth soccer athletes from Bahia de Feira - BA

\begin{abstract}
This work deals with the implantation of a living pharmacy in the Higher Education Unit of Feira de Santana - UNEF with the intent of using medicinal plants with therapeutic characteristics related to the sports activities of young soccer players, both for preventive and curative purposes. The living pharmacy allows to apply the popular culture and scientific use of plants in the treatment of minor injuries or health maintenance that happen in this type of sport. For the implantation, a wooden structure in the shape of an octagonal mandala was built for planting the 8 selected species: alecrim (Rosmarinus officinalis L.), arnica (Porophyllum ruderale (Jacq.) Cass.), babosa (Aloe vera (L.) Burn.f), calendula (Calendula officinalis L.), chicória (Cichorium intybus L.), mastruz (Dysphania ambrosioides (L.) Mosyakin and Clemants), Plantago major L. (mastruz) and terramicina (Alternanthera brasiliana (L.) Kuntze), being arranged in a triangular format for each specimen and deposited in the UNEF Green Garden for cultivation for the intent of using in athletes according to their sporting needs. Thus, throughout this initiative, the implementation of living pharmacy proved to be a highly viable system for the sport, from cultivation to the use of medicinal plants that help maintain health or prevent minor injuries, as well as being a alternative drug therapy based on herbal medicines, which consequently allows the reduction of expenses with synthetic medicines.
\end{abstract}

\section{Introdução}

O uso de plantas para fins medicinais é uma prática comum disseminada entre diversas culturas no mundo todo, tradicionalmente passada de geração em geração. Durante anos,

\footnotetext{
*Autor correspondente: luiz_farmaco@ hotmail.com (da Rocha J.L.C.)
} 
as plantas representaram o principal recurso médico no tratamento de diversas enfermidades e única fonte de matéria-prima na fabricação de medicamentos. Com a modernização, surgiram os medicamentos sintéticos e parte dessa tradição foi perdida principalmente devido à falta de segurança com a comprovação das propriedades terapêuticas das plantas. Entretanto, as novas tendências globais de uma preocupação com a biodiversidade e as ideias de desenvolvimento sustentável despertaram novamente o interesse geral na fitoterapia, resultando no estabelecimento de novas linhas de pesquisa nas universidades brasileiras, a fim de buscar bases mais sólidas para a validação científica do uso de plantas medicinais (1).

Ao final da década de 70, a Organização Mundial de Saúde passou a reconhecer o uso de fitoterápicos e desde então tem expressado através de comunicados e resoluções a importância do uso desses medicamentos no âmbito sanitário. Tendo em vista que mais de $80 \%$ da população mundial utiliza produtos à base de plantas medicinais nos cuidados da saúde pelo acesso oficial de cultura popular (seja ela indígena, quilombola e outras) como terapia alternativa, a OMS tem recomendado aos seus estados-membros a elaboração de políticas públicas integrativas que insiram o uso de fitoterápicos no sistema de saúde (2).

Nos últimos anos, com o avanço dos estudos científicos, a fitoterapia ganhou papel de destaque como alternativa terapêutica, a partir de então, surgiu a ideia de criar a Farmácia Viva, um modelo de sistema realizado em etapas, através do cultivo, colheita, conservação e utilização de plantas medicinais que são selecionadas por sua eficácia e segurança terapêutica e validadas cientificamente. A denominação "Farmácia Viva" surgiu no ano de 1983, no estado do Ceará, a partir do trabalho acadêmico do Prof. Dr. Francisco José de Abreu Matos, na Universidade Federal do Ceará, tendo como modelo as recomendações da Organização Mundial de Saúde (OMS) (3).

No Brasil, mais especificamente na região Nordeste, o acesso ao sistema de saúde ainda é bastante limitado, principalmente por parte da população mais carente, fator que favorece a essa mesma parcela da população a recorrer a terapias alternativas, como o uso de plantas medicinais no tratamento de diferentes doenças. E diante dessa demanda sociocultural, muitas cidades brasileiras passaram a implantar a Farmácia Viva a fim de promover o acesso de forma correta e segura das plantas medicinais a população $(4,5)$.

Recentemente, a cidade de Lauro de Freitas-Ba, implantou a Farmácia viva visando a dedicação do estudo dessas plantas para interpretá-las cientificamente e passar o conhecimento para a comunidade. Essa utilização das plantas medicinais aproxima o conhecimento cultural, étnico e científico (4). Tais fatores favorecem sua utilização com maior ênfase por parte da população carente como forma de medicina alternativa (6).

Diante do exposto, o objetivo do trabalho foi a implantação da Farmácia Viva na Unidade de Ensino Superior de Feira de Santana - UNEF, a fim de, promover o uso seguro e racional de plantas medicinais através da aplicabilidade da fitoterapia nos jogadores do Bahia de Feira, para fins preventivos e curativos, disponibilizando plantas medicinais a comunidade desportiva de acordo com suas necessidades.

\section{Materiais e Métodos}

A implantação da Farmácia Viva foi feita na Horta Green UNEF, localizada na Unidade de Ensino Superior de Feira de Santana (UNEF), na cidade de Feira de Santana, Bahia, Brasil, em parceria com a Associação Desportiva Bahia de Feira na cidade de Feira de Santana-BA.

\subsection{Levantamento do uso de plantas medicinais}


Foi realizada uma análise através de artigos científicos que abordassem os agravos acometidos a categoria desportiva a respeito dos possíveis problemas de saúde em atletas juvenis de futebol, a partir do perfil socioeconômico. Tendo em vista que a grande parte é de baixa renda, estão mais suscetíveis a doenças provenientes da falta de saneamento básico, as infecções parasitárias, como também problemas comuns aos atletas decorrente da rotina de treino como: lesões musculares por contato ou intensidade dos jogos e treinos, contusões, impacto direto com outros jogadores, infecções fúngicas de pele, entre outras.

A partir desses dados, foi feita a seleção de plantas medicinais baseadas no uso tradicional popular e que possuem validação científica, comprovando suas propriedades terapêuticas que visam a contribuir na manutenção da saúde dos atletas, com isso, sanando possíveis enfermidades.

\subsection{Definição das plantas medicinais cultivadas na UNEF}

Parte das plantas utilizadas para a criação da Farmácia Viva foi doada pela população, por diferentes cidades da região da Bahia. A arnica foi obtida na cidade de Riachão do Jacuípe-Ba. Por outro lado, babosa, mastruz e tanchagem foram doados pela população da cidade de Feira de Santana-Ba, e a terramicina pela população de Bom Jesus dos Pobres-Ba. Mudas de alecrim, chicória e calêndula foram compradas em horto comercial da cidade de Feira de Santana-Ba.

Após as aquisições, todas as plantas foram transferidas para a Horta Green da UNEF, sendo as 8 espécies identificadas e confirmadas seus táxons com base no uso de chave de identificação, pela botânica Wilma Brandão de Andrade, e revisadas a nomenclatura botânica a nível de espécie através http://www.theplantlist.org/.

As plantas foram selecionadas levando em consideração, principalmente, sua ação fitoterápica, assim como a fácil adaptação às condições climáticas da região de Feira de Santana-Ba.

Após a validação cientifica com base nas propriedades fitoterápicas de interesse, foram escolhidas 8 plantas medicinais na construção da Farmácia Viva alecrim (Rosmarinus officinalis L.), arnica (Porophyllum ruderale (Jacq.) Cass.), babosa (Aloe vera (L.) Burm.f), calêndula (Calendula officinalis L.), chicória (Cichorium intybus L.), mastruz (Dysphania ambrosioides (L.) Mosyakin e Clemants), tanchagem (Plantago major L.) e terramicina (Alternanthera brasiliana (L.) Kuntze).

Em seguida, foram feitas replicatas dessas espécies e mantidas em local apropriado na Horta Green UNEF com luminosidade adequada para o crescimento das mudas até o dia do plantio definitivo (7).

\subsection{Implantação da farmácia viva}

Para a implantação do canteiro, foi escolhido o modelo de mandala, com um círculo octogonal construído com material de reflorestamento, tendo como medidas 2 metros de comprimento, circunferência de $2 \mathrm{~m}^{2}$ e altura de $15 \mathrm{~cm}$. Para a separação das plantas e setorização das mesmas, foram dispostos 8 espaços em formato triangular com 1 metro de comprimento cada um. 


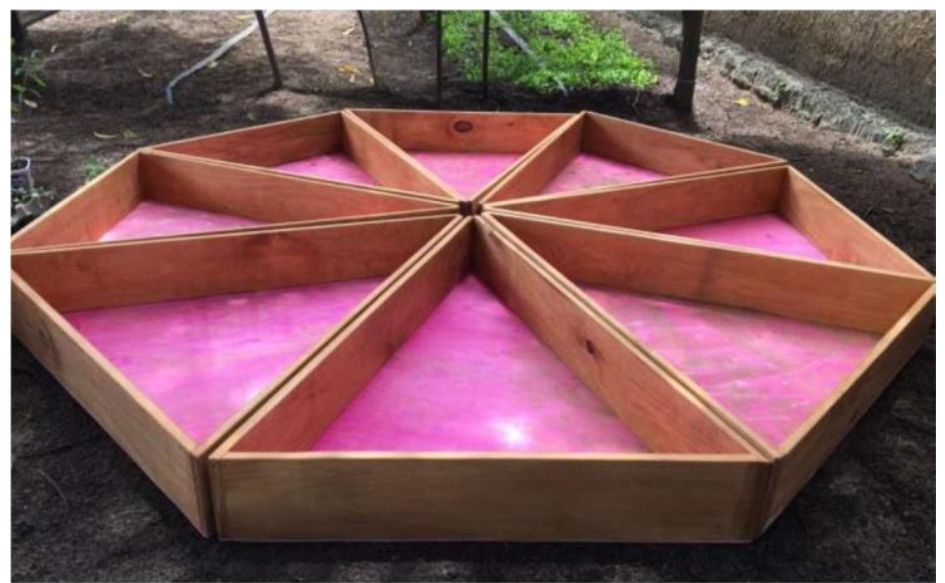

Figura 1 - Modelo de mandala construída em formato octogonal com setorização para cada espécime das plantas medicinais.

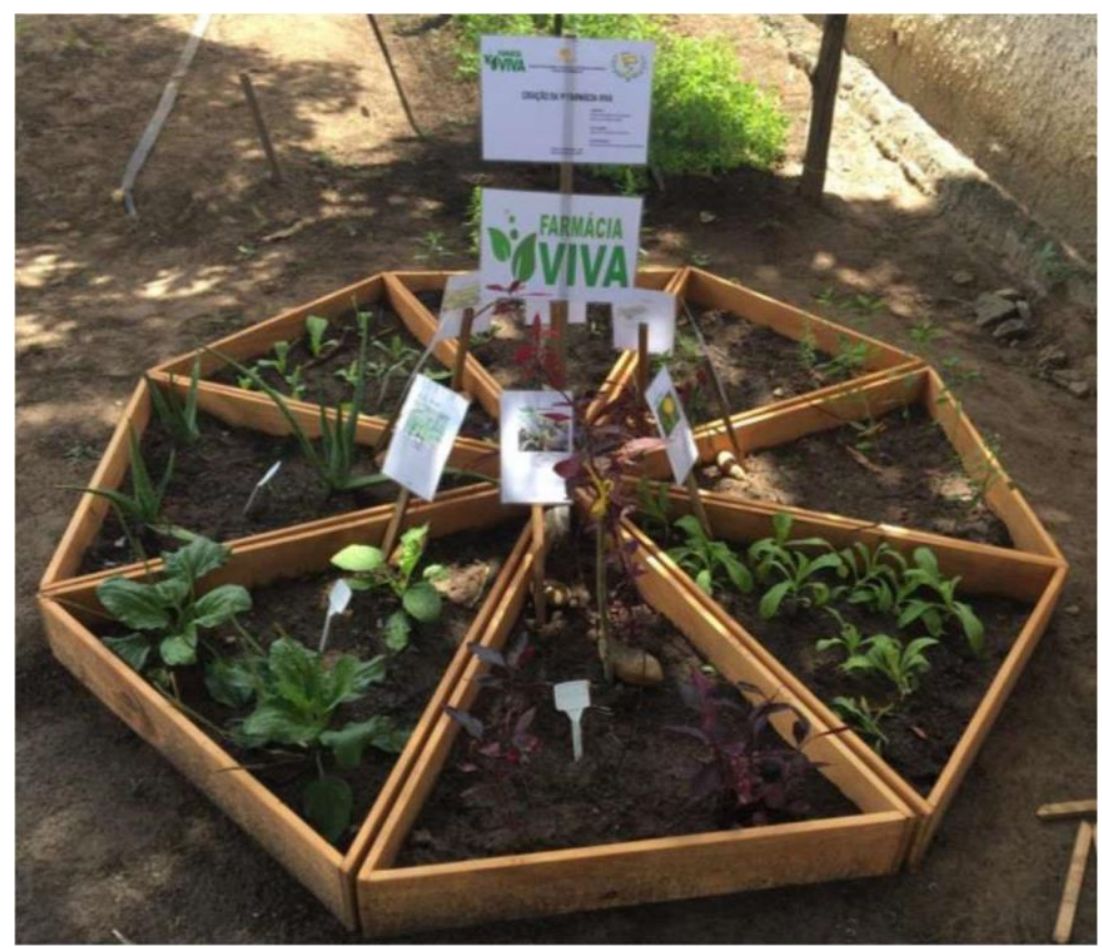

Figura 2 - Canteiro finalizado com as 8 espécies de interesse fitoterápico para jogadores do Bahia de Feira com placas contendo algumas informações, fotografia da planta adulta para facilitar a identificação de cada espécie na hora da coleta.

Os espaçamentos entre as plantas foram de $20 \mathrm{~cm}$ e profundidade de $5 \mathrm{~cm}$, porém algumas plantas possuem uma densidade característica específica onde foi necessário espaçamento de $10 \mathrm{~cm}$ (Figura 2).

Após o plantio, para facilitar o uso ou manejo por parte da comunidade acadêmica, foi criado um manual com as indicações terapêuticas das plantas medicinais selecionadas e com as respectivas informações: nome popular, espécie, uso, parte utilizada, condições de plantio e cultivo e observações.

\section{Resultados e discussão}

Em 1983, através dos estudos acadêmicos feitos pelo Prof. Dr. Francisco José de Abreu 
Matos na Universidade Federal do Ceará, surge o conceito de "Farmácias Vivas", que, a partir desses estudos, passa a ter a medicina tradicional com preparações caseiras e a produção ou plantação de plantas medicinais em pequenos locais. Para que haja seu uso, essas plantas devem ser cientificamente identificadas e validadas com comprovação científica da sua efetividade em um horto, localizado junto às escolas de farmácia ou de saúde pública da região ou centros de ciências da saúde de universidades (8).

O conceito básico sobre farmácia viva é o de sempre ter plantas medicinais de fácil acesso e baixo custo, ao alcance das mãos, com indicações no tratamento de sintomas e doenças mais comuns e de menor agravo. Nesse sentido, apresenta-se como peça-chave de um modelo nacional que abrange desde a questão cultural até a socioeconômica da população envolvida, contribuindo para a preservação, valorização do conhecimento tradicional e popular sobre o uso de plantas medicinais (9).

Em 2010, o Ministério da Saúde brasileiro através da portaria $\mathrm{n}^{\circ} 2.960$, passa a inserir o Programa Nacional de Plantas Medicinais e Fitoterápicos no Sistema Único de Saúde (SUS), como forma de reconhecimento e valorização da cultura tradicional de plantas medicinais no país. Uma vez instituído no SUS, o Programa Nacional de Plantas Medicinais e Fitoterápicos tem por finalidade garantir a população brasileira a segurança, a eficácia e a qualidade das plantas medicinais e fitoterápicos aplicando o uso das plantas através das farmácias vivas (nativas e não nativas) com o intuito de sanar enfermidades e doenças de pequeno agravo da população. farmacológica $(10,11,12)$.

Com o levantamento do uso de plantas medicinais para o tratamento de pequenas enfermidades, é possível conhecer suas propriedades curativas associadas ao consumo que é confirmado através dos conhecimentos científico e cultural tradicional, que contribuem para o uso adequado das plantas $(13,14)$.

Com base nos estudos científicos (11) e conhecimento empírico sobre a indicações do uso dessas plantas medicinais voltadas para as necessidades que acometem esses atletas de futebol, pode-se observar no Quadro 1 as oito espécies selecionadas para a implantação da farmácia viva.

Quadro 1 - Espécie de Plantas Medicinais presentes na mandala implantada na Horta Green UNEF.

\begin{tabular}{|l|l|l|}
\hline $\begin{array}{c}\text { Nomenclatura } \\
\text { Vernacular }\end{array}$ & \multicolumn{1}{|c|}{ Nomenclatura Botânica } & \multicolumn{1}{|c|}{ Indicações } \\
\hline Arnica do mato & $\begin{array}{l}\text { Porophyllum ruderale } \\
\text { (Jacq.) Cass.** }\end{array}$ & $\begin{array}{l}\text { Anti-inflamatório, analgésico tópico (traumatismos, } \\
\text { entorses, artrites, nevralgias, hematomas) antialérgico, } \\
\text { antibacteriano, antimicótico. Lesões secundárias a } \\
\text { contusões, distensões musculares, dores reumáticas } \\
(17) .\end{array}$ \\
\hline Alecrim & Rosmarinus officinalis L.* & $\begin{array}{l}\text { Distúrbios circulatórios, antisséptico e cicatrizante, } \\
\text { dispepsia (distúrbios digestivos) (18). }\end{array}$ \\
\hline Babosa & Aloe Vera (L.) Burm.f $*$ & $\begin{array}{l}\text { Antibacteriana, cicatrizante para queimadura de } 1^{\circ} \\
\text { grau, antivirótica e antifúngica (19). }\end{array}$ \\
\hline Calêndula & Calendula officinalis L.* & $\begin{array}{l}\text { Espasmódica, expectorante, anti-inflamatória, } \\
\text { cicatrizante de feridas e queimaduras e das mucosas } \\
\text { buco-faríngea, dor de garganta, alergia e icterícia; } \\
(20) .\end{array}$ \\
\hline
\end{tabular}




\begin{tabular}{|l|l|l|}
\hline $\begin{array}{c}\text { Nomenclatura } \\
\text { Vernacular }\end{array}$ & \multicolumn{1}{|c|}{ Nomenclatura Botânica } & \multicolumn{1}{|c|}{ Indicações } \\
\hline Chicória & Cichorium intybus L.* & $\begin{array}{l}\text { Anti-inflamatório das dores musculares e nas } \\
\text { articulações ou na prática de exercícios físicos com } \\
\text { frequência. (21). }\end{array}$ \\
\hline Mastruz & $\begin{array}{l}\text { Dysphania ambrosioides (L.) } \\
\text { Mosyakin e Clemants* }\end{array}$ & $\begin{array}{l}\text { Vermífuga, estomáquica, cicatrizante, sudorífica, } \\
\text { antiséptica tópica, diurética, tônica, antiulcerosa, } \\
\text { antifúngica, sedativa, carminativa, anticancerígena, } \\
\text { purgante, eupéptica, antigripal, emoliente, } \\
\text { antiasmática, antiespasmódica, anti-inflamatória (1). }\end{array}$ \\
\hline Tanchagem & Plantago major L.* & $\begin{array}{l}\text { Adstringentes, descongestionantes, emolientes, contra } \\
\text { as diarréias, hemorragias, afecções pulmonares e e } \\
\text { bronquiais (18). }\end{array}$ \\
\hline Terramicina & $\begin{array}{l}\text { Alternanthera brasiliana (L.) } \\
\text { Kuntze* }\end{array}$ & $\begin{array}{l}\text { Analgésicas e anti-inflamatórias, antibiótica, } \\
\text { diurética, depurativa, adstringente, antiviral, } \\
\text { antidiarreica, tosse e gripe (1). }\end{array}$ \\
\hline
\end{tabular}

Fonte: Farmacopeia Brasileira, 2010 (19)*; Duarte et al., 2007 (17)** e Manual de Plantas Medicinais.

O trabalho científico executado com os jogadores juvenis de futebol, teve um papel de destaque com a estruturação do espaço por meio de demandas históricas dos relatos dos jogadores de futebol, junto com estudos etnobotânicos sobre o uso de plantas medicinais que indicam, dentre outros elementos, como responsáveis pela popularização do uso das plantas medicinais na manutenção ou no tratamento de doenças de pequenos agravos (18).

Devido ao fato de o futebol ser um esporte que exige muito esforço físico é inevitável que as lesões durante sua prática aconteçam, visto que é caracterizado por intenso contato físico, movimentos curtos, rápidos e não contínuos, tais como aceleração, desaceleração, saltos e mudanças abruptas de direção, quedas, entre outras (16). Desta forma, o uso de determinadas plantas medicinais contribui de forma significativa na manutenção da saúde desses atletas expostos a esses eventos.

Com base no estudo realizado por Silva e colaboradores (14), corroboram que o uso da terapia medicamentosa a partir de plantas medicinais e fitoterápicos apontam uma melhora dos atletas em $68 \%$ em relação a intensidade da dor referida por esses desportistas devido ao efeito e a atividade terapêutica comprovadas cientificamente.

Após a estruturação e implantação da Farmácia Viva, foi realizada uma palestra para a comunidade acadêmica e desportiva do Bahia de Feira, a fim de, apresentar-lhes o novo sistema e informar sobre as suas vantagens e possibilidades, orientando-os quanto aos critérios que deverão ser seguidos. Para melhor esclarecimento, foram distribuídas cartilhas informativas sobre os tipos de plantas que estão sendo cultivadas, quais suas indicações, formas de cultivo, modo de utilização e possíveis contraindicações

\section{Conclusão}

A implantação da Farmácia Viva na UNEF se mostrou um sistema de grande viabilidade por permitir que os jogadores de futebol do Bahia de Feira tivessem fácil acesso as plantas medicinais, impactando de forma positiva na qualidade de vida desses atletas, através de um tratamento constante na prevenção de agravos de doenças, manutenção, promoção, e recuperação da saúde do indivíduo, orientação do uso correto das plantas medicinais e também que em relação aos gastos com medicamentos sintéticos, o uso da fitoterapia, passa a ser um sistema de baixo custo financeiro, e que possibilita a redução de gastos.

Por ser a primeira Farmácia Viva criada com o objetivo inicial de atender as necessidades terapêuticas dos atletas juvenis de futebol, faz-se necessário o incentivo à 
expansão da Farmácia Viva a outras modalidades que viabilizem a produção dos insumos terapêuticos a partir das plantas e até a fabricação de produtos fitoterápicos elevando o sistema a um outro patamar.

Com a iniciativa desse trabalho, a Farmácia Viva permite que a comunidade acadêmica expanda seus conhecimentos através da pesquisa desde o cultivo até a utilização de plantas medicinais, como também na seleção botânica terapêutica mais indicada na manutenção da saúde dos atletas do Bahia de Feira, podendo ser ampliado na região para toda a comunidade através de parcerias com a atenção básica de saúde.

\section{Agradecimentos}

Este trabalho desenvolveu-se com apoio da Unidade de Ensino Superior de Feira de Santana - UNEF e a Associação Desportiva do Bahia de Feira - ADBF.

\section{Referências}

1. Lorenzi H, Matos FJA. Plantas Medicinais no Brasil: nativas e exóticas. Nova Odessa: Instituto Plantarum de Estudos de Flora. 2009; 512.

2. Ministério da Saúde. Secretaria de Atenção à Saúde. Departamento de Atenção Básica. Práticas integrativas e complementares: plantas medicinais e fitoterapia na Atenção Básica/Ministério da Saúde. Secretaria de Atenção à Saúde. Departamento de Atenção Básica. - Brasília: Ministério da Saúde. 2012; 156 p.: il. - (Série A. Normas e Manuais Técnicos) (Cadernos de Atenção Básica; n. 31).

3. Matos FJA. O projeto Farmácias-Vivas e a fitoterapia no nordeste do Brasil. Revista de Ciências Agroveterinárias 2009; 5(01): 24-32.

4. Bianchi RV. Farmácia da natureza: um modelo eficiente de farmácia viva. 2016; 10.

5. Pinto JDM, Nascimento WMC, Oliveira MAS. Perfil das prescrições de fitoterápicos atendidas no Centro de Saúde da Família "Cleide Cavalcante", Sobral -CE, Brasil, 2006; 176-182.

6. Gonçalves R. GD Gzeta Digital. A importância de farmácias vivas. [Online].; 2016 [cited 20180529. Disponível em https://www.gazetadigital.com.br/editorias/opiniao/a-importancia-defarmaciasvivas $/ 496007$

7. Souza VC, Lorenzi H. Botânica sistemática: guia ilustrado para identificação das famílias de fanerógamas nativas e exóticas no Brasil, baseado em APG II. 2008; 2 ed. Nova Odessa: Instituto Plantarum, 703.

8. Lorenzi H, Martinsa RD, Pintobo RH, Sennab SM, Limar AWS, De Carvalho CRF, Fontesd DAMF, Barrose FA, Ximenes RM. Estruturação do Espaço Farmácia Viva na Universidade Federal de Pernambuco como Estratégia para Formação em Fitoterapia. Vittalle - Revista de Ciências da Saúde 2018; 30(1): 182-191.

9. Randal VB; Behrens M, Pereira AMS. Nature pharmacy: an effective model of living pharmacy. Revista Fitos 2016; 10(1): 1-93.

10. Ministério da Saúde. Portaria n ${ }^{\circ} 886$, de 20 de abril de 2010. Institui a Farmácia Viva no âmbito do Sistema Único de Saúde. Disponível em http://www.brasilsus.com.br/legislacoes/gm/103778-886?q=. Acesso em 11 de março de 2019.

11. Ministério da Saúde. (MS). Política e Programa Nacional de Plantas Medicinais e Fitoterápicos. 2016; ed. 1, p.17-18.

12. Ministério da Saúde. Portaria ${ }^{\circ} 886$, de 20 de abril de 2010. Institui a Farmácia Viva no âmbito do Sistema Único de Saúde. Disponível em http://www.brasilsus.com.br/legislacoes/gm/103778-886?q= . Acesso em 11 de março de 2019.

13. Amorozo MCM. Uso e diversidade de plantas medicinais em Santo Antônio do Leverger, MT, Brasil. Acta Botanica Brasilica. 2002; 16 (2):189-203.

14. Silva ALA, Cangueiro BL, Silva CBA, Santos GSP, Peniche GG, Oliveira CR, Souza VMA. Avaliação de produto fitoterápico de uso tópico na dor musculoesquelética em ginastas. Brazilian Journal of Natural Sciences 2018; 1(1): 805-825.

15. Martins RD, Pinto RH, Senna SM, Lima AWS, Mota CRFC, Fontes DAF, Barros FA, Ximenes RM. 
Estruturação do Espaço Farmácia Viva na Universidade Federal de Pernambuco como Estratégia para Formação em Fitoterapia. Vittalle - Revista de Ciências da Saúde 2018; 20(1):182-191.

16. Almeida PSM, Scotta AP, Pimentel BM, Batista Júnior S, Sampaio YR. Incidência De Lesão Musculoesquelética Em Jogadores De Futebol. Revista Brasileira Medicina Esporte 2013; 19(2): 112115.

17. Duarte MR, Siebenrok MCN, Empinotti CB. Anatomia comparada de espécies de arnica: Porophyllum ruderale (Jacq.) Cass. e Chaptalia nutans (L.) Pohl. Revista Ciências Farmaceutica Básica Aplicada 2007; 28(2):193-201.

18. Grandi TSM. Tratado das plantas medicinais: mineiras, nativas e cultivadas. 1 ed. Adaequatio estúdio 2014; 461-462.

19. Anvisa - Agência Nacional de Vigilância Sanitária Fundação Oswaldo Cruz. Farmacopeia Brasileira 2010; 5(2).

20. Muñoz J, Machado JEM, González CMT. Validación de una metodología por HPLC para cuantificar quercetina total en extractos de Calendula officinalis. Revista Cubana de Farmácia 2015; 49(1): 91102.

21. Rocha MQ, Noda H, Noda SN. Avaliação agronômica de procedências de "chicória" (Eryngium foetidum L.) com ênfase nas populações do alto rio solimões. Xl Jornada de Iniciação Científica do PIBICl INPA 2002; 168-169. 